\title{
Microbial Diversity in the Rhizosphere of Some Wild Plants in Riyadh Region, Saudi Arabia
}

\author{
Fahad Nasser AL-Barakah*, Fahd Abdulla Saleh Alnohait, Muhammad Sohaib \\ Muhammad Ramzan and Samir Radwan
}

\author{
Soil Sciences Department, College of Food and Agriculture Sciences, King Saud University, \\ P. O. Box 2460, Riyadh, 11451, Saudi Arabia
}

*Corresponding author

\begin{tabular}{l}
\hline Ke y w o r d s \\
$\begin{array}{l}\text { Deiversity, } \\
\text { Microorgaisms, } \\
\text { Meadows, } \\
\text { Rhizosphere, } \\
\text { Wild Plants }\end{array}$ \\
\hline Article Info \\
$\begin{array}{l}\text { Accepted: } \\
\text { 20 September } 2020 \\
\text { Available Online: } \\
\text { 10 October } 2020\end{array}$ \\
\hline
\end{tabular}

\section{A B S T R A C T}

Four meadows were selected in Riyadh region namely, Kherarh, Al-Masoudi, ShoaibHarimlae and Al-Khabia for this study. Nine wild plants from each meadow were chosen from herbs, shrubs and trees to carry out this work. The obtained results showed that densities of total microbial counts were generally higher in rhizosphere of different wild plants, compared to control (bara) soil. The total microbial counts in the rhizosphere regions of Calotropis procera, Hamada elegans, Lycium shawii and Rhayza stricta plants were relatively higher than those in the rhizosphere of the other studied wild plants. The higher total fungi count in the rhizosphere were observed in Ziziphus snummularia and Calotropis procera plants growing in Al-Masoudi meadow, while the lower total fungi counts were recorded in the rhizosphere of Trigonella anguina growing plant in Al-Khabiah meadow. Azotobacter spp. occurred in higher densities in rhizosphere of Hamada elegans and Tripleurospermum auriculatum plants in Al-Khabiah and Al-Kherarh meadows, respectively. The higher Azospirillum spp. counts were observed in Tripleurospermum auriculatum, Trigonella anguina, Hamada elegans and Acacia gerrardii plants growing in Shoaib Harimlae meadow, while the lower Azospirillum spp. counts were recorded in the rhizosphere of Rhayza stricta in the same meadow. Phosphate dissolving bacteria counts in the rhizosphere of Acacia gerrardii and Lycium shawii plants in Al-Khabiah and Al-Kherarh meadows, respectively exceeded those found in the rhizosphere of other wild plants under different meadows. Pseudomonas sp. occurred in higher densities in rhizosphere of Acacia gerrardii, Ziziphus snummularia and Rhayza stricta in Shoaib Harimlae meadow. The higher Streptomyces spp. counts observed in the rhizosphere Tripleurospermum auriculatum and Launaea capitata plants growing in Al-Khabiah meadow. Cellulose decomposing bacteria counts in the rhizosphere of Tripleurospermum auriculatum, Launaea capitata and Rhayza stricta plants in Al-Khabiah meadow and Calotropis procera in Shoaib Harimlae meadow exceeded those found in the rhizosphere of other wild plants under different meadows.

\section{Introduction}

Wild plants are numerous and have their own characteristics in combating desertification improving the local climate, fixing sand dunes, conserving soils, preventing erosion and flood damages, producing forage and other benefits. They are very important economically, environmentally, and medicinally. Therefore, it is of importance to 
conserve these plants on a sustainable use basis where research and development are of main concern to improve and to diversify them. Biodiversity is essential for environmental and economical use of these wild plants. Wild plants in Riyadh region of Saudi Arabia tend to grow and live as individuals or in groups with similar characteristics such as the halophytic vegetation (Tamarix, Salsola, Suaeda, Zygophyllum), sandy vegetation (Haloxylon, Lepladenia, Calligonium) rocky and wadi vegetation (Ziziphus, Maerua, Capparis, Acacia, Lycium) and others as mentioned by Al- Nohat (2007).

Soil is a highly complex and variable matrix comprising a wide range of habitat and supporting some of the most species-rich, biochemically diverse and microbial communities in nature (Abd El-Fattah et al., 2011). The activity and diversity of soil microbial communities fluctuate in response to alterations in the environmental conditions (Steele and Streit, 2006). Many microorganisms live in soil, but even more live close to the roots of plants (Amal et al., 2003, Majjami, 2020).

The rhizosphere is an important site of microbial activity in desert soils, since it provides ample carbon substrate in arid soil which are poor in organic matter. There are different types of substances that diffuse from the roots and that stimulate the microbial activity, such as carbohydrates (sugars and oligosaccharides), organic acids, vitamins, nucleotides, flavonoids, enzymes, hormones, and volatile compounds (Prescott et al., 1999). The rhizosphere or the zone of influence around plant roots harbors a multitude of microorganisms that are affected by both abiotic and biotic stresses.

The extent of the rhizosphere varies with the plant and the soil, but it is widely accepted that it covers at least $2 \mathrm{~mm}$ from the rhizoplane (Vega, 2007). The release of root exudates can be affected by several factors related to plant, soil and environment. According to Bowen and Rovira (1999), plants can release between $10-30 \%$ of photosynthates through the root system.

Microorganisms play a key role in nutrient cycling by decomposing and mineralizing organic material and releasing as well as transforming inorganic nutrients. Additionally, microorganisms can affect plant growth and nutrient uptake by release of growth-stimulating or -inhibiting substances that influence root physiology and root system architecture (Govindasamy et al., 2009, Marschner et al., 2011).

Growth and activity of soil microorganisms are mainly limited by carbon availability (De Nobili et al., 2001 and Demoling et al., 2007). Hence, the release of exudates by roots results in higher microbial density and metabolic activity in the rhizosphere than the bare soil (Gomes et al., 2001, Berg et al., 2002), which reflects the selective enrichment of different populations depending on amount and composition of root exudates. Exudate amount and composition vary among plant species and along the root axis, and are further modified in response to plant phenology, nutrient status, environmental stresses and diseases (Neumann, 2007).

Sunantapongsuk (2003) reported that microbial populations and activities are higher in the vetiver rhizosphere than outside of the rhizosphere. There are several beneficial rhizo-microorganisms in the rhizosphere, which can improve soil quality, enhance crop production and protection, conserve natural resources and ultimately create more sustainable agricultural production and safe environment (Vaddar, 2007). 
Saudi Arabian soil is infested with many soil borne fungi, 25 genera and 68 species, in addition to one variety of each of Aspergillus chevalieri, Aspergillus flavus and Aspergillus nidulans were isolated from 40 soil samples collected from desert in Saudi Arabia on $5 \%$ sodium chloride-Czapek agar (Abdel-Hafez 1981). Altalhi (2004) recorded fifteen fungal species belonging to ten genera from the rhizosphere of some plants from AL-Taif region. Abou-Zeid et al., (2011) in their study collected and identified twenty two wild plants from Taif Governorate. Pathogenic fungi were isolated from some of these plants and identified as Alternaria alternate, Ulocladium botrytis, Cladosporium spp., Cephalosporium spp., Penicillium chrysogenum, Fusarium oxysporum and Humicola grisea. Sheikh (2010) isolated 18 bacterial and 5 fungal species from soil samples collected from El-Madina, Saudi Arabia. Study of Abd El-Fattah et al., (2011) in Taif, the number of fungal population on the rhizosphere of Launae sonchoides was 562 colony/g, while it was 469 colonies/gm of dry soil in the rhizosphere of Artemisia princeps. They also isolated ten different fungal genera from the rhizosphere of each of the two plants, the genus Aspergillus sp. was the most frequent followed by Monilia, Rhizoctonia and Rhizopus.

The present study had been carry out on selected local plants that usually found as wild flora in some meadows of Riyadh region. Diversity and densities of different microorganisms present in the rhizosphere of selected wild plants growing in these meadows were determined.

\section{Materials and Methods}

\section{Screening and collection plant types}

During the period from March to April 2018, common plant types were screen end and collected from some meadows in Riyadh region and characterized in the diversity of plant types and differences in environmental conditions. Four meadows were selected in Riyadh region namely are: Al-Kherarh, AlMasoudi, ShoaibHarimlae and Al-Khabiah. Nine wild plants from each meadow were choosen from herbs, shrubs and trees to carry out this study, and three replicates of each selected types. All plant species were identified at herbarium of College of Science, King Saud University. Selected wild plants were Tripleurospermum auriculatum, Trigonella anguina, Launaea capitate, Rhayza stricta, Hamada elegans, Lycium shawii, Acacia gerrardii, Ziziphus nummularia, and Calotropis procera. The nine wild plants names and their families, genera and species of each meadow are given in table 1 .

\section{Description of the selected meadows}

Al-Kherarh meadow is located in the southwest of Riyadh (N 24- 23. $820^{\circ}$, E 46.'. $14^{-} .760^{\circ}$ ). This meadow is of large space, located in the bosom Nfod Guenivzh southern province of Muzahimiyah. Wild spread plants in this meadow were Acacia, Ziziphus, Lycium, Calotropis and some perennials plants and seasonal herbs. AlKhabiah meadow is located north of Hawta BaniTamim, east Soat Valley (N 23-. 4.745 ${ }^{\circ}$, $\mathrm{E} 46^{\circ}$. 56. $420^{\circ}$ ). This meadow is medium space. It is characterized by the types of many trees. The torrent is coming from the bottom of Mansaf before flooding in the valley, in addition to whip coral descend from the surrounding mountains. Wild spread plants in this meadow were Acacia, Ziziphus, Acacia ehrenbergiana, Lycium, Calotropis and some perennials plants and seasonal herbs. Shoaib Harimlae is broad plain, land gravel, especially the belly of the valley ( $\mathrm{N} \mathrm{25}$. $04^{-}$. $068^{0}$, E $046^{-} .03^{-} .113^{0}$ ) contains large Acacia trees. It is considered a national park in Saudi 
Arabia due to the length of valleys and complexity of trees and dense vegetation. AlMasoudi meadow is in the north-east of the Riyadh city, (N 25- $12^{-} .00^{\circ}$, E 47- $27^{-} .00^{\circ}$ ). It is very large space and pours in the Valley Masudi. Wild spread plants in this meadow were Acacia, Ziziphus, Lycium, Rhanterium, Rhayza, Calotropis and herbs.

\section{Collection of rhizosphere soil samples}

Soil samples from rhizosphere of selected plants were collected, as well as, collecting soil samples from plant-free soil (bare soil) for each meadows as control. Counts of many microorganisms were determined in rhizosphere soil samples. Specific media were used to determination counts of microorganisms. The serial dilution plate method or the most probable number method were used for counting total microbial counts on nutrient agar medium; total fungi count on Martin's Medium; Azotobacter sp. on modified Ashby's medium (Abd El-Malek and Ishac, 1968); Azospirillum sp. on semisolid malate medium (Dobereiner et al., 1976); phosphate dissolving bacteria on modified Bunt and Rovira medium (Abd ElHafez, 1966); Pseudomonus sp. on KB medium (Sands and Rovira, 1970) and Streptomyces sp. on Jensen's medium.

\section{Determination of soil physical and chemical characteristics}

From the abovementioned meadows 40 composite surface soil samples $(0-30 \mathrm{~cm})$ were collected under the different plant species beside the bare soils in each meadow. The collected soil samples were air dried thoroughly mixed and crushed to pass through a $2 \mathrm{~mm}$ sieve and stored for the chemical and physical analysis of $\mathrm{pH}, \mathrm{EC}$ using a $\mathrm{pH}-$ meter according to Thomas, (1996) while the EC values were determined in soil paste extract using the EC-meter according to Rhodes
(1996). The chemical composition of the studied samples was determined according to Rainwater and Thatcher, (1979) for the determination of soluble $\mathrm{SO}_{4}{ }^{2-}$, Sparks et al., (1996) for the determinations of soluble $\mathrm{Na}^{+}$, $\mathrm{K}^{+}, \mathrm{Ca}^{2+}, \mathrm{Mg}^{2+}, \mathrm{HCO}_{3}{ }^{-}, \mathrm{CO}_{3}{ }^{2-}$ andCl ${ }^{-}$. The values of sodium adsorption ratio ( $\mathrm{SAR}$ ) was also calculated

On the other hand, calcium carbonate contents in the soil samples were determined as in the method described by Loeppert and Suarez (1996). Particle size distributions was determined according to Gee and Bauder (1996). On the other hand, the soil organic matter (O.M) content was determined according to Nelson and Sommers, (1996). Also, the available concentrations of N, P, K, $\mathrm{Fe}, \mathrm{Cu}, \mathrm{Zn}$ and $\mathrm{Mn}$ in soil samples were determined as described by George et al., (2013). The extractions content of the studied metals ( $\mathrm{Fe}, \mathrm{Cu}, \mathrm{Zn}$ and $\mathrm{Mn}$ ) in the solutions were determined by the ICP (Perkin Elmer, Model 4300 DV).

\section{Results and Discussion}

\section{Soil Physical and Chemical Properties}

The basic physical and chemical properties of the studied soil samples are statistically summarized in Tables (2 and 3). The texture class of soil samples ranged from loam or sandy loam, to loamy sand in most cases. The overall mean values of sand, silt and clay contents in the collected soils were 65,22 and $13 \%$, respectively, regardless of meadow location and/or growing plant species. The studied soils were calcareous in nature $\left(29.5 \% \mathrm{CaCO}_{3}\right.$ in average). On the other hand the soils were poor in their organic matter content especially in bare soils $(0.16 \%$ in average), however, the soil O.M. contents were relatively higher (from two to seven times compered to bare soils) affecting either with the meadow location and/or the growing plant species. 
On the other hand, the soils having mean $\mathrm{pH}$ values of 7.7, and SAR values of 2.6 with an approximately $\mathrm{EC}_{\mathrm{e}}$ values of $1.03 \mathrm{dS} . \mathrm{m}^{-1}$, respectively, regardless off meadow location and/or the growing plant species. In such soils the $\mathrm{Na}^{+}, \mathrm{Ca}^{2+}$ and, $\mathrm{Mg}^{2+}$ ions were the most dominant cations, while the $\mathrm{Cl}^{-}$and $\mathrm{SO}_{4}{ }^{2-}$ ions were the most dominant anions.

This means that, the lack in rainfall in such area has led to increase the concentration of salts as well as total carbonate in surface soil layers. Moreover, the lack of rainfall has also led to reduced vegetation cover in bare soils in each meadow, reflecting the low soil organic matter content. These properties also reflect the fact that the soils of the studied meadows were virgin and had not been subjected to any activities which affect their properties.

Data presented in table (4) clearly revealed that the chemical properties of the studied soils were affected by the growing plant species regardless of the studied meadow. Obviously soil $\mathrm{pH}$ values were reduced in the areas covered with plant as compared with the uncovered soils, as the Hamada elegans plants were most effective in this respect. In contrast, the soil salinity values were increased in the areas covered by plants except for Ziziphus nummularia and $T$. onguina. This may be due to that the root exudate from the growing plants resulted in reducing soil $\mathrm{pH}$ values.

In this respect, Marschner (1995) pointed out that the rhizosphere $\mathrm{pH}$ is usually lower than the bare soil in 1-2 units due to several mechanisms which are responsible of this effect such as production of $\mathrm{CO}_{2}$ by respiration processes, or by pump of $\mathrm{H}^{+}$in nutrient uptake by plant and microbes, or the release of organic acids by roots and microbes.
Available soil nutrient content as affected by plant species and meadow location

Data presented in table (5) indicated that the nutrient content of the studied soils $(\mathrm{Cu}, \mathrm{Fe}$, $\mathrm{Mn}, \mathrm{Zn}, \mathrm{N}, \mathrm{P}$ and $\mathrm{K}$ ) were affected favorably either by the growing plant species and meadow location. Generally, the nutrients in studied soils were adequate for the available $\mathrm{Cu}, \mathrm{Fe}, \mathrm{Mn}, \mathrm{N}$, and $\mathrm{P}$ while it was marginal for available $\mathrm{Zn}$ and low for available $\mathrm{K}$ according to the classification given by George et al., (2013).

With respect to the role of the growing plant species on nutrients availability data presented in Table (5) clearly appear that the nutrient concentrations of the studied soils in the studied areas were affected favorably by the growing plant species regardless of the studied meadow. The soils of Trigonella anguina plants having relatively higher contents of $\mathrm{Cu}, \mathrm{Fe}, \mathrm{Mn}, \mathrm{Zn}, \mathrm{N}$ and $\mathrm{P}$ as the rate of increment in such nutrients reached 150,260, 433, 240, 167 and $256 \%$ over their content in the bare soil, respectively.The effects can vary with the soil buffer capacity and the type of plant species. as mentioned before the $\mathrm{pH}$ values were redused as a result of root exudate therefore, the acid conditions favor the solubilization of soil minerals (Bowen and Rovira 1999), as well as increasing the availability of micronutrients.

Regarding the role of meadow location in soil nutrient concentrations data presented in Table (5) showed that the nutrient concentrations in the studied areas were affected favorably by meadow location regardless of the growing plant species. The soils of Al-Khabiah meadow having relatively higher available $\mathrm{Cu}, \mathrm{Fe}, \mathrm{Mn}, \mathrm{Zn}, \mathrm{P}$ and $\mathrm{K}$ as well as higher soil organic matter content $(1.264 \%)$ compared either to the bare soil and the other studied three meadows. 
Table.1 Tested wild plants in some meadows of Riyadh region

\begin{tabular}{|l|c|c|}
\hline \multicolumn{1}{|c|}{ Scientific Name of plant } & Family & Type plant \\
\hline Tripleurospermum auriculatum & Asteraceae & Herb \\
\hline Trigonella anguina & Papillionaceae & Herb \\
\hline Launaea capitate & Asteraceae & Herb \\
\hline Rhayza stricta & Apocynaceae & Shrub \\
\hline Hamada elegans & Henopodiaceae & Shrub \\
\hline Lycium shawii & Solanaceae & Shrub \\
\hline Acacia gerrardii & Mimosaceae & Tree \\
\hline Ziziphus nummularia & Rhamnaceae & Shrub \\
\hline Calotropis procera & Asclepiadaceae & Tree \\
\hline
\end{tabular}

Table.2 Soil Chemical analysis of the studied meadows under different plant species

\begin{tabular}{|c|c|c|c|c|c|c|c|c|c|c|c|c|c|}
\hline \multirow[t]{2}{*}{ Meadow } & \multirow[t]{2}{*}{ Type of Plant } & \multirow[t]{2}{*}{ pH } & \multirow{2}{*}{$\begin{array}{c}E C \\
(\mathbf{d S} / \mathbf{m})\end{array}$} & \multicolumn{4}{|c|}{ Cations(meq/l) } & \multicolumn{4}{|c|}{ Anions(meq/l) } & \multirow[t]{2}{*}{ SAR } & \multirow{2}{*}{$\begin{array}{c}\text { OM } \\
\%\end{array}$} \\
\hline & & & & $\mathrm{Ca}^{2+}$ & $\mathrm{Mg}^{2+}$ & $\mathrm{Na}^{+}$ & $\mathrm{K}^{+}$ & $\mathrm{CO}_{3}{ }^{2-}$ & $\mathrm{HCO}_{3}^{-}$ & $\mathrm{Cl}^{-}$ & $\mathrm{SO}_{4}{ }^{2-}$ & & \\
\hline \multirow{10}{*}{ 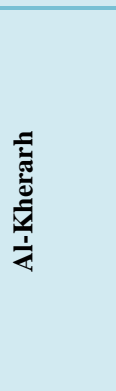 } & Bare soil & 7.91 & 1.5 & 6.14 & 1.77 & 6.81 & 0.21 & 0.00 & 1.48 & 7.41 & 5.99 & 3.43 & 0.14 \\
\hline & $\begin{array}{l}\text { Tripleurospermum } \\
\text { auriculatum }\end{array}$ & 7.65 & 0.5 & 2.05 & 0.59 & 2.27 & 0.07 & 0.00 & 0.49 & 2.47 & 2.00 & 1.98 & 0.76 \\
\hline & Launaea capitata & 7.66 & 0.5 & 2.05 & 0.59 & 2.27 & 0.07 & 0.00 & 0.49 & 2.47 & 2.00 & 1.98 & 0.55 \\
\hline & Trigonella anguina & 7.72 & 0.75 & 3.07 & 0.88 & 3.41 & 0.11 & 0.00 & 0.74 & 3.70 & 2.99 & 2.42 & 0.62 \\
\hline & Hamada elegans & 7.47 & 0.75 & 3.07 & 0.88 & 3.41 & 0.11 & 0.00 & 0.74 & 3.70 & 2.99 & 2.42 & 0.41 \\
\hline & Lycium shawii & 7.35 & 1.25 & 5.12 & 1.47 & 5.68 & 0.18 & 0.00 & 1.24 & 6.17 & 4.99 & 3.13 & 1.31 \\
\hline & Rhayza stricta & 7.4 & 1.75 & 7.17 & 2.06 & 7.95 & 0.25 & 0.00 & 1.73 & 8.64 & 6.98 & 3.70 & 0.48 \\
\hline & Calotropis procera & 7.73 & 0.75 & 3.07 & 0.88 & 3.41 & 0.11 & 0.00 & 0.74 & 3.70 & 2.99 & 2.42 & 0.62 \\
\hline & Ziziphus nummularia & 7.22 & 0.75 & 3.07 & 0.88 & 3.41 & 0.11 & 0.00 & 0.74 & 3.70 & 2.99 & 2.42 & 0.69 \\
\hline & Acacia gerrardii & 7.67 & 0.5 & 2.05 & 0.59 & 2.27 & 0.07 & 0.00 & 0.49 & 2.47 & 2.00 & 1.98 & 0.97 \\
\hline \multirow{10}{*}{$\begin{array}{l}\frac{a}{\pi} \\
\frac{\pi}{\frac{\pi}{\pi}} \\
\frac{\pi}{\frac{\pi}{4}} \\
\frac{1}{4}\end{array}$} & Bare soil & 7.96 & 0.5 & 2.05 & 0.59 & 2.27 & 0.07 & 0.00 & 0.49 & 2.47 & 2.00 & 1.98 & 0.28 \\
\hline & $\begin{array}{l}\text { Tripleurospermum } \\
\text { auriculatum }\end{array}$ & 7.78 & 0.5 & 2.05 & 0.59 & 2.27 & 0.07 & 0.00 & 0.49 & 2.47 & 2.00 & 1.98 & 2 \\
\hline & Launaea capitata & 7.82 & 0.5 & 2.05 & 0.59 & 2.27 & $\mathbf{0 . 0 7}$ & 0.00 & 0.49 & 2.47 & 2.00 & 1.98 & 1.24 \\
\hline & Trigonella anguina & 7.81 & 0.5 & 2.05 & 0.59 & 2.27 & 0.07 & 0.00 & 0.49 & 2.47 & 2.00 & 1.98 & 0.9 \\
\hline & Hamada elegans & 7.52 & 0.25 & 1.02 & 0.29 & 1.14 & 0.04 & 0.00 & 0.25 & 1.23 & 1.00 & 1.40 & 1.38 \\
\hline & Lycium shawii & 7.68 & 0.25 & 1.02 & 0.29 & 1.14 & 0.04 & 0.00 & 0.25 & 1.23 & 1.00 & 1.40 & 1.73 \\
\hline & Rhayza stricta & 7.74 & 0.75 & 3.07 & 0.88 & 3.41 & 0.11 & 0.00 & 0.74 & 3.70 & 2.99 & 2.42 & 2.21 \\
\hline & Calotropis procera & 7.51 & 0.5 & 2.05 & 0.59 & 2.27 & 0.07 & 0.00 & 0.49 & 2.47 & 2.00 & 1.98 & 0.55 \\
\hline & Ziziphus nummularia & $\mathbf{7 . 5 8}$ & 0.5 & 2.05 & 0.59 & 2.27 & 0.07 & 0.00 & 0.49 & 2.47 & 2.00 & 1.98 & 0.9 \\
\hline & Acacia gerrardii & 7.64 & 0.5 & 2.05 & 0.59 & 2.27 & 0.07 & 0.00 & 0.49 & 2.47 & 2.00 & 1.98 & 1.45 \\
\hline \multirow{10}{*}{ 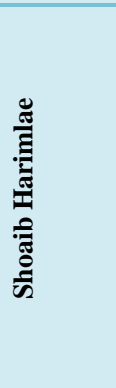 } & Bare soil & 8.25 & 0.25 & 1.02 & 0.29 & 1.14 & 0.04 & 0.00 & 0.25 & 1.23 & 1.00 & 1.40 & 0.14 \\
\hline & $\begin{array}{l}\text { Tripleurospermum } \\
\text { auriculatum }\end{array}$ & 7.46 & 3.25 & 13.31 & 3.83 & 14.77 & 0.46 & 0.00 & 3.21 & 16.05 & 12.97 & 5.04 & 0.69 \\
\hline & Launaea capitata & 7.43 & 4.25 & 17.41 & 5.01 & 19.31 & 0.60 & 0.00 & 4.20 & 20.98 & 16.96 & 5.77 & 0.62 \\
\hline & Trigonella anguina & 7.52 & 0.75 & 3.07 & 0.88 & 3.41 & 0.11 & 0.00 & 0.74 & 3.70 & 2.99 & 2.42 & 1.04 \\
\hline & Hamada elegans & 7.54 & 2.25 & 9.21 & 2.65 & 10.22 & 0.32 & 0.00 & 2.22 & 11.11 & 8.98 & 4.20 & 0.69 \\
\hline & Lycium shawii & 7.77 & 0.75 & 3.07 & 0.88 & 3.41 & 0.11 & 0.00 & 0.74 & 3.70 & 2.99 & 2.42 & 0.62 \\
\hline & Rhayza stricta & 7.47 & 6.25 & 25.60 & 7.37 & 28.40 & 0.88 & 0.00 & 6.18 & 30.86 & 24.94 & 6.99 & 0.69 \\
\hline & Calotropis procera & 7.56 & 1 & 4.10 & 1.18 & 4.54 & 0.14 & 0.00 & 0.99 & 4.94 & 3.99 & 2.80 & 0.76 \\
\hline & Ziziphus nummularia & 7.9 & 0.5 & 2.05 & 0.59 & 2.27 & 0.07 & 0.00 & 0.49 & 2.47 & 2.00 & 1.98 & 0.83 \\
\hline & Acacia gerrardii & 7.55 & 1.25 & 5.12 & 1.47 & 5.68 & 0.18 & 0.00 & 1.24 & 6.17 & 4.99 & 3.13 & 1.38 \\
\hline \multirow{10}{*}{ 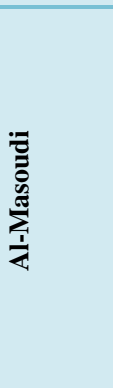 } & Bare soil & 8.07 & 0.25 & 1.02 & 0.29 & 1.14 & 0.04 & 0.00 & 0.25 & 1.23 & 1.00 & 1.40 & 0.07 \\
\hline & $\begin{array}{l}\text { Tripleurospermum } \\
\text { auriculatum }\end{array}$ & 7.72 & 0.75 & 3.07 & 0.88 & 3.41 & 0.11 & 0.00 & 0.74 & 3.70 & 2.99 & 2.42 & 0.55 \\
\hline & Launaea capitata & 7.49 & 1.75 & 7.17 & 2.06 & 7.95 & 0.25 & 0.00 & 1.73 & 8.64 & 6.98 & 3.70 & 0.97 \\
\hline & Trigonella anguina & 7.59 & 0.25 & 1.02 & 0.29 & 1.14 & 0.04 & 0.00 & 0.25 & 1.23 & 1.00 & 1.40 & 1.59 \\
\hline & Hamada elegans & 7.66 & 1.5 & 6.14 & 1.77 & 6.81 & 0.21 & 0.00 & 1.48 & 7.41 & 5.99 & 3.43 & 0.97 \\
\hline & Lycium shawii & 7.6 & 0.75 & 3.07 & 0.88 & 3.41 & 0.11 & 0.00 & 0.74 & 3.70 & 2.99 & 2.42 & 1.59 \\
\hline & Rhayza stricta & 7.75 & 0.25 & 1.02 & 0.29 & 1.14 & 0.04 & 0.00 & 0.25 & 1.23 & 1.00 & 1.40 & 0.69 \\
\hline & Calotropis procera & 7.58 & 0.75 & 3.07 & 0.88 & 3.41 & 0.11 & 0.00 & 0.74 & 3.70 & 2.99 & 2.42 & 0.69 \\
\hline & Ziziphus nummularia & 7.62 & 0.25 & 1.02 & 0.29 & 1.14 & 0.04 & 0.00 & 0.25 & 1.23 & 1.00 & 1.40 & 0.48 \\
\hline & Acacia gerrardii & 7.75 & 0.5 & 2.05 & 0.59 & 2.27 & 0.07 & 0.00 & 0.49 & 2.47 & 2.00 & 1.98 & 0.62 \\
\hline
\end{tabular}


Table.3 Soil Physical properties of the studied meadows under different plant species

\begin{tabular}{|c|c|c|c|c|c|c|}
\hline \multirow[t]{2}{*}{ Meadow } & \multirow[t]{2}{*}{ Type of Plant } & \multicolumn{3}{|c|}{ Partical Size distrebution \% } & \multirow[t]{2}{*}{ Texture class } & \multirow[t]{2}{*}{$\mathrm{CaCO}_{3}(\%)$} \\
\hline & & Clay & Silt & Sand & & \\
\hline \multirow{10}{*}{ 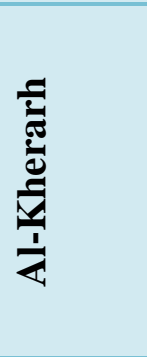 } & Bare soil (Control) & 22.5 & 34.0 & 43.5 & loam & 19.00 \\
\hline & Tripleurospermum auriculatum & 6.5 & 16.0 & 77.5 & loamy sand & 12.40 \\
\hline & Launaea capitata & 26.5 & 30.0 & 43.5 & loam & 13.00 \\
\hline & Trigonella anguina & 27.5 & 33.0 & 39.5 & clay loam & 22.00 \\
\hline & Hamada elegans & 8.5 & 4.0 & 87.5 & loamy sand & 4.80 \\
\hline & Lycium shawii & 18.5 & 22.0 & 59.5 & sandy loam & 7.20 \\
\hline & Rhayza stricta & 35.5 & 37.0 & 27.5 & clay loam & 12.20 \\
\hline & Calotropis procera & 32.5 & 42.0 & 25.5 & clay loam & 22.00 \\
\hline & Ziziphus nummularia & 28.5 & 38.0 & 33.5 & clay loam & 15.60 \\
\hline & Acacia gerrardii & 10.5 & 24.0 & 65.5 & sandy loam & 12.00 \\
\hline \multirow{10}{*}{ 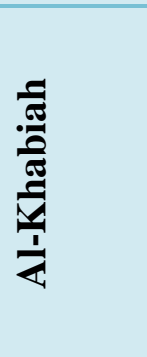 } & Bare soil (Control) & 9.5 & 15.0 & 75.5 & sandy loam & 56.60 \\
\hline & Tripleurospermum auriculatum & 12.5 & 60.0 & 27.5 & silt loam & 33.00 \\
\hline & Launaea capitata & 9.5 & 25.0 & 65.5 & sandy loam & 40.00 \\
\hline & Trigonella anguina & 21.5 & 33.0 & 45.5 & loam & 34.80 \\
\hline & Hamada elegans & 26.5 & 56.0 & 17.5 & silt loam & 52.00 \\
\hline & Lycium shawii & 19.5 & 27.0 & 53.5 & sandy loam & 25.60 \\
\hline & Rhayza stricta & 16.5 & 22.0 & 61.5 & sandy loam & 34.40 \\
\hline & Calotropis procera & 22.5 & 32.0 & 45.5 & loam & 41.00 \\
\hline & Ziziphus nummularia & 20.5 & 30.0 & 49.5 & loam & 41.40 \\
\hline & Acacia gerrardii & 14.5 & 24.0 & 61.5 & sandy loam & 36.00 \\
\hline \multirow{10}{*}{ 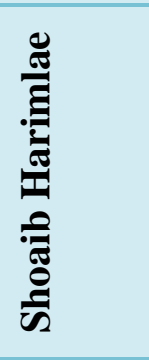 } & Bare soil (Control) & 5.0 & 4.0 & 90.9 & sand & 68.20 \\
\hline & Tripleurospermum auriculatum & 1.0 & 16.1 & 82.9 & loamy sand & 56.40 \\
\hline & Launaea capitata & 5.0 & 8.0 & 86.9 & loamy sand & 40.20 \\
\hline & Trigonella anguina & 3.0 & 14.1 & 82.9 & loamy sand & 34.40 \\
\hline & Hamada elegans & 6.0 & 7.0 & 86.9 & loamy sand & 54.00 \\
\hline & Lycium shawii & 9.1 & 6.0 & 84.9 & loamy sand & 57.60 \\
\hline & Rhayza stricta & 1.0 & 12.1 & 86.9 & sand & 85.00 \\
\hline & Calotropis procera & 3.0 & 12.1 & 84.9 & loamy sand & 73.20 \\
\hline & Ziziphus nummularia & 3.0 & 16.1 & 80.9 & loamy sand & 34.60 \\
\hline & Acacia gerrardii & 6.0 & 19.1 & 74.8 & sandy loam & 39.40 \\
\hline \multirow{10}{*}{ 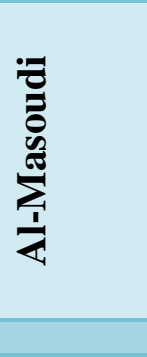 } & Bare soil (Control) & 1.0 & 4.0 & 95.0 & sand & 8.80 \\
\hline & Tripleurospermum auriculatum & 6.0 & 13.1 & 80.9 & loamy sand & 14.60 \\
\hline & Launaea capitata & 7.0 & 16.1 & 76.9 & sandy loam & 15.60 \\
\hline & Trigonella anguina & 9.1 & 24.1 & 66.8 & sandy loam & 20.00 \\
\hline & Hamada elegans & 16.1 & 29.2 & 54.7 & sandy loam & 5.20 \\
\hline & Lycium shawii & 5.0 & 24.1 & 70.8 & sandy loam & 15.40 \\
\hline & Rhayza stricta & 7.0 & 12.1 & 80.9 & loamy sand & 3.80 \\
\hline & Calotropis procera & 17.1 & 18.1 & 64.8 & sandy loam & 4.60 \\
\hline & Ziziphus nummularia & 15.1 & 12.1 & 72.8 & sandy loam & 7.20 \\
\hline & Acacia gerrardii & 9.1 & 6.0 & 84.9 & loamy sand & 6.80 \\
\hline
\end{tabular}

Table.4 Impact of plant species on soil properties regardless of meadow location

\begin{tabular}{|c|c|c|c|c|c|c|c|c|c|c|c|c|}
\hline \multirow[t]{2}{*}{ Type of Plant } & \multirow[t]{2}{*}{ pH } & \multirow{2}{*}{$\begin{array}{c}\mathrm{EC} \\
(\mathrm{dS} / \mathrm{m})\end{array}$} & \multicolumn{4}{|c|}{ Cations(meq/l) } & \multicolumn{4}{|c|}{ Anions(meq/l) } & \multirow[t]{2}{*}{ SAR } & \multirow{2}{*}{$\underset{\%}{\text { OM }}$} \\
\hline & & & $\mathrm{Ca}^{2+}$ & $\mathrm{Mg}^{2+}$ & $\mathrm{Na}^{+}$ & $\mathrm{K}^{+}$ & $\mathrm{CO}_{3}{ }^{2-}$ & $\mathrm{HCO}_{3}{ }^{-}$ & $\mathrm{Cl}^{-}$ & $\mathrm{SO}_{4}^{2-}$ & & \\
\hline Bare soil (Control) & 8.0 & 0.6 & 2.6 & 0.7 & 2.8 & 0.1 & 0.0 & 0.6 & 3.1 & 2.5 & 2.1 & 0.2 \\
\hline $\begin{array}{l}\text { Tripleurospermum } \\
\text { auriculatum }\end{array}$ & 7.7 & 1.3 & 5.1 & 1.5 & 5.7 & 0.2 & 0.0 & 1.2 & 6.2 & 5.0 & 2.9 & 1.0 \\
\hline Launaea capitata & 7.6 & 1.8 & 7.2 & 2.1 & 8.0 & 0.2 & 0.0 & 1.7 & 8.6 & 7.0 & 3.4 & 0.8 \\
\hline Trigonella anguina & 7.7 & 0.6 & 2.3 & 0.7 & 2.6 & 0.1 & 0.0 & 0.6 & 2.8 & 2.2 & 2.1 & 1.0 \\
\hline Hamada elegans & 7.5 & 1.2 & 4.9 & 1.4 & 5.4 & 0.2 & 0.0 & 1.2 & 5.9 & 4.7 & 2.9 & 0.9 \\
\hline Lycium shawii & 7.6 & 0.8 & 3.1 & 0.9 & 3.4 & 0.1 & 0.0 & 0.7 & 3.7 & 3.0 & 2.3 & 1.3 \\
\hline Rhayza stricta & 7.6 & 2.3 & 9.2 & 2.7 & 10.2 & 0.3 & 0.0 & 2.2 & 11.1 & 9.0 & 3.6 & 1.0 \\
\hline Calotropis procera & 7.6 & 0.8 & 3.1 & 0.9 & 3.4 & 0.1 & 0.0 & 0.7 & 3.7 & 3.0 & 2.4 & 0.7 \\
\hline Ziziphus nummularia & 7.6 & 0.5 & 2.0 & 0.6 & 2.3 & 0.1 & 0.0 & 0.5 & 2.5 & 2.0 & 1.9 & 0.7 \\
\hline Acacia gerrardii & 7.7 & 0.7 & 2.8 & 0.8 & 3.1 & 0.1 & 0.0 & 0.7 & 3.4 & 2.7 & 2.3 & 1.1 \\
\hline
\end{tabular}


Table.5 Available nutrients contentin the studied meadow under different plant species

\begin{tabular}{|c|c|c|c|c|c|c|c|c|}
\hline \multirow[t]{2}{*}{ Meadow } & \multirow{2}{*}{ Type of Plant } & \multicolumn{7}{|c|}{ Available macro and micro nutrients (mg/kg) } \\
\hline & & Cu & $\mathbf{F e}$ & Mn & $\mathrm{Zn}$ & $\mathbf{N}$ & $\mathbf{P}$ & $\mathbf{K}$ \\
\hline \multirow{10}{*}{ 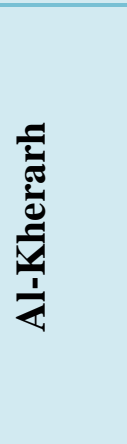 } & Bare soil (Control) & 0.37 & 6.02 & 2.38 & 0.38 & 42.0 & 1.0 & 24.3 \\
\hline & Tripleurospermum auriculatum & 0.48 & 10.62 & 10.66 & 1.00 & 77.0 & 8.2 & 13.3 \\
\hline & Launaea capitata & 0.65 & 13.59 & 9.86 & 1.07 & 42.0 & 2.2 & 6.1 \\
\hline & Trigonella anguina & 0.46 & 8.97 & 8.37 & 0.80 & 77.0 & 12.0 & 18.0 \\
\hline & Hamada elegans & 0.17 & 6.07 & 3.77 & 0.48 & 112.0 & 3.5 & 21.7 \\
\hline & Lycium shawii & 0.45 & 8.59 & 13.21 & 1.64 & 115.5 & 8.2 & 23.0 \\
\hline & Rhayza stricta & 0.22 & 8.75 & 2.68 & 0.54 & 49.0 & 14.0 & 7.1 \\
\hline & Calotropis procera & 0.41 & 10.79 & 9.36 & 0.93 & 59.5 & 3.5 & 14.5 \\
\hline & Ziziphus nummularia & 0.42 & 7.56 & 9.17 & 1.06 & 94.5 & 6.0 & 18.0 \\
\hline & Acacia gerrardii & 1.57 & 10.61 & 12.20 & 2.32 & 73.5 & 8.9 & 18.0 \\
\hline \multirow{10}{*}{ 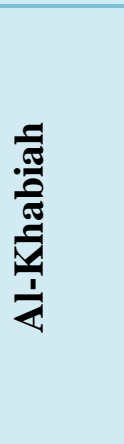 } & Bare soil (Control) & 0.66 & 7.20 & 3.45 & 0.63 & 42.0 & 0.4 & 25.6 \\
\hline & Tripleurospermum auriculatum & 0.98 & 17.07 & 24.84 & 3.30 & 77.0 & 9.5 & 24.3 \\
\hline & Launaea capitata & 1.30 & 21.90 & 10.98 & 1.45 & 77.0 & 9.1 & 72.6 \\
\hline & Trigonella anguina & 1.39 & 24.96 & 10.26 & 1.52 & 129.5 & 8.9 & 35.6 \\
\hline & Hamada elegans & 1.07 & 6.51 & 2.33 & 0.68 & 66.5 & 9.2 & 23.0 \\
\hline & Lycium shawii & 1.13 & 19.92 & 11.81 & 2.10 & 81.5 & 9.6 & 28.4 \\
\hline & Rhayza stricta & 0.91 & 19.98 & 20.44 & 2.42 & 77.0 & 8.2 & 21.7 \\
\hline & Calotropis procera & 1.05 & 7.31 & 2.09 & 0.95 & 42.0 & 1.2 & 43.5 \\
\hline & Ziziphus nummularia & 1.32 & 16.48 & 6.82 & 1.81 & 59.5 & 13.6 & 24.3 \\
\hline & Acacia gerrardii & 0.94 & 14.23 & 11.44 & 2.26 & 59.5 & 14.2 & 18.0 \\
\hline \multirow{10}{*}{ 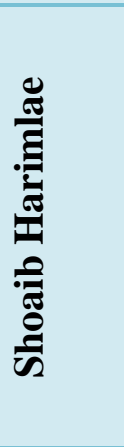 } & Bare soil (Control) & 0.27 & 5.29 & 2.53 & 0.34 & 42.0 & 0.3 & 6.1 \\
\hline & Tripleurospermum auriculatum & 0.47 & 9.71 & 7.01 & 0.86 & 94.5 & 20.2 & 35.6 \\
\hline & Launaea capitata & 0.42 & 10.35 & 7.98 & 1.12 & 112.0 & 22.1 & 45.1 \\
\hline & Trigonella anguina & 0.63 & 21.40 & 11.59 & 1.19 & 77.0 & 17.7 & 14.5 \\
\hline & Hamada elegans & 0.47 & 7.68 & 6.54 & 1.16 & 115.5 & 3.9 & 14.5 \\
\hline & Lycium shawii & 0.24 & 5.91 & 4.22 & 0.46 & 59.5 & 13.5 & 19.2 \\
\hline & Rhayza stricta & 0.30 & 6.50 & 4.82 & 0.57 & 143.5 & 3.9 & 18.0 \\
\hline & Calotropis procera & 0.32 & 7.15 & 5.33 & 0.79 & 59.5 & 6.8 & 34.1 \\
\hline & Ziziphus nummularia & 0.74 & 6.66 & 5.97 & 0.43 & 24.5 & 14.5 & 11.2 \\
\hline & Acacia gerrardii & 1.02 & 14.43 & 22.66 & 1.62 & 59.5 & 0.5 & 24.3 \\
\hline \multirow{10}{*}{ 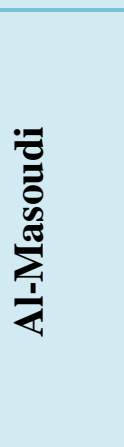 } & Bare soil (Control) & 0.18 & 7.70 & 2.90 & 0.63 & 42.0 & 0.3 & 8.1 \\
\hline & Tripleurospermum auriculatum & 0.89 & 19.43 & 12.19 & 1.51 & 59.5 & 10.7 & 25.6 \\
\hline & Launaea capitata & 0.96 & 17.36 & 10.82 & 1.54 & 59.5 & 18.9 & 21.7 \\
\hline & Trigonella anguina & 1.66 & 39.62 & 29.42 & 3.33 & 164.5 & 32.6 & 31.2 \\
\hline & Hamada elegans & 0.27 & 7.44 & 5.87 & 0.95 & 129.5 & 15.7 & 23.0 \\
\hline & Lycium shawii & 1.04 & 17.25 & 14.02 & 1.78 & 24.5 & 20.3 & 43.5 \\
\hline & Rhayza stricta & 0.17 & 8.76 & 3.96 & 0.41 & 42.0 & 12.0 & 10.1 \\
\hline & Calotropis procera & 0.74 & 15.46 & 11.43 & 1.29 & 59.5 & 9.0 & 34.1 \\
\hline & Ziziphus nummularia & 0.61 & 40.22 & 8.31 & 0.91 & 59.5 & 0.9 & 15.6 \\
\hline & Acacia gerrardii & 0.54 & 31.76 & 5.13 & 0.69 & 42.0 & 8.2 & 15.6 \\
\hline
\end{tabular}


Table.6 Total counts of microbes in the rhizosphere of wild plants in some meadows of Riyadh region

\begin{tabular}{|c|c|c|c|c|}
\hline \multirow[t]{2}{*}{ Type of plant } & Al Kherarh & Al-Masoudi & $\begin{array}{c}\text { Shoaib } \\
\text { Harimlae }\end{array}$ & Al-Khabiah \\
\hline & \multicolumn{4}{|c|}{ CFU X $10^{5}$} \\
\hline Control (bare soil) & 1.1 & 1.2 & 1.3 & 1.3 \\
\hline Tripleurospermum auriculatum & 30 & 61 & 142 & 59 \\
\hline Trigonella anguina & 129 & 62 & 145 & 39 \\
\hline Launaea capitata & 153 & 81 & 196 & 101 \\
\hline Rhayza stricta & 30 & 89 & 376 & 341 \\
\hline Hamada elegans & 287 & 393 & 305 & 27 \\
\hline Lycium shawii & 124 & 196 & 603 & 53 \\
\hline Acacia gerrardii & 166 & 128 & 192 & 154 \\
\hline Ziziphus nummularia & 48 & 79 & 401 & 164 \\
\hline Calotropis procera & 126 & 228 & 776 & 130 \\
\hline
\end{tabular}

Table.7 Counts of total fungi in the rhizosphere of wild plants in some meadows of Riyadh region

\begin{tabular}{|c|c|c|c|c|}
\hline \multirow[t]{2}{*}{ Type of plant } & Al Kherarh & Al-Masoudi & $\begin{array}{c}\text { Shoaib } \\
\text { Harimlae }\end{array}$ & Al-Khabiah \\
\hline & \multicolumn{4}{|c|}{ CFU X $10^{2}$} \\
\hline Control (bare soil) & 10 & 20 & 20 & 12 \\
\hline Tripleurospermum auriculatum & 170 & 120 & 180 & 60 \\
\hline Trigonella anguina & 10 & 60 & 20 & 20 \\
\hline Launaea capitata & 40 & 180 & 60 & 90 \\
\hline Rhayza stricta & 20 & $\mathbf{5 0}$ & 70 & 20 \\
\hline Hamada elegans & 40 & 120 & 30 & 40 \\
\hline Lycium shawii & 30 & 120 & 60 & 40 \\
\hline Acacia gerrardii & 30 & 60 & 70 & 60 \\
\hline Ziziphus nummularia & 90 & 680 & 20 & 40 \\
\hline Calotropis procera & 30 & 530 & 180 & 20 \\
\hline
\end{tabular}

Table.8 Counts of Azotobacter sp. in the rhizosphere of wild plants in some meadows of Riyadh region

\begin{tabular}{|c|c|c|c|c|}
\hline \multirow[t]{2}{*}{ Type of plant } & Al-Kherarh & Al-Masoudi & $\begin{array}{c}\text { Shoaib } \\
\text { Harimlae }\end{array}$ & Al-Khabiah \\
\hline & \multicolumn{4}{|c|}{ CFU X 10 ${ }^{3}$} \\
\hline Control (bare soil) & 4.4 & 3 & 4 & 2 \\
\hline Tripleurospermum auriculatum & 103 & 12 & 43 & 43 \\
\hline Trigonella anguina & 64 & 13 & 26 & 40 \\
\hline Launaea capitata & 76 & 36 & 30 & 78 \\
\hline Rhayza stricta & 47 & 8 & 10 & 40 \\
\hline Hamada elegans & 34 & 13 & 10 & 106 \\
\hline Lycium shawii & 55 & 13 & 9 & 79 \\
\hline Acacia gerrardii & 92 & 8 & 10 & 40 \\
\hline Ziziphus nummularia & 90 & 14 & 9 & 39 \\
\hline Calotropis procera & 88 & 21 & 7 & 30 \\
\hline
\end{tabular}


Table.9 Counts of Azospirillum sp. in the rhizosphere of wild plants in some meadows of Riyadh region

\begin{tabular}{|c|c|c|c|c|}
\hline \multirow[t]{2}{*}{ Type of plant } & Al-Kherarh & Al-Masoudi & Shoaib Harimlae & Al-Khabiah \\
\hline & \multicolumn{4}{|c|}{ CFU X 10 3} \\
\hline Control (bare soil) & 1.1 & 2.2 & 1.5 & 1.3 \\
\hline Tripleurospermum auriculatum & 12.2 & 15 & 16 & 11.3 \\
\hline Trigonella anguina & 11 & 13 & 16.5 & 12.5 \\
\hline Launaea capitata & 16 & 9.2 & 9.2 & 1.7 \\
\hline Rhayza stricta & 5.4 & 5.4 & 1.8 & 1.49 \\
\hline Hamada elegans & 1.4 & 3.5 & 16 & 1.95 \\
\hline Lycium shawii & 3.5 & 5.4 & 2.8 & 2.6 \\
\hline Acacia gerrardii & 10.8 & 11.5 & 16 & 2.6 \\
\hline Ziziphus nummularia & 2.8 & 5.4 & 3.5 & 2.8 \\
\hline Calotropis procera & 16 & 14.5 & 2.4 & 2.2 \\
\hline
\end{tabular}

Table.10 Counts of phosphate dissolving bacteria in the rhizosphere of wild plants in some meadows of Riyadh region

\begin{tabular}{|c|c|c|c|c|}
\hline \multirow[t]{2}{*}{$\begin{array}{c}\text { Type of } \\
\text { plant }\end{array}$} & Al Kherarh & Al-Masoudi & $\begin{array}{c}\text { Shoaib } \\
\text { Harimlae }\end{array}$ & Al-Khabiah \\
\hline & \multicolumn{4}{|c|}{ CFU X 10 ${ }^{4}$} \\
\hline Control (bare soil) & 1.1 & 1.2 & 2.3 & 3 \\
\hline Tripleurospermum auriculatum & 13 & 21 & 30 & 26 \\
\hline Trigonella anguina & 17 & 8 & 32 & 7 \\
\hline Launaea capitata & 24 & 6 & 24 & 16 \\
\hline Rhayza stricta & 8 & 18 & 8 & 14 \\
\hline Hamada elegans & 20 & 12 & 12 & 17 \\
\hline Lycium shawii & 95 & 9 & 10 & 52 \\
\hline Acacia gerrardii & 28 & 22 & 24 & 101 \\
\hline Ziziphus nummularia & 16 & 17 & 10 & 9 \\
\hline Calotropis procera & 103 & 16 & 26 & 5 \\
\hline
\end{tabular}

Table.11 Counts of Pseudomonas sp. in the rhizosphere of wild plants in some meadows of Riyadh region

\begin{tabular}{|c|c|c|c|c|}
\hline \multirow[t]{2}{*}{ Type of plant } & Al Kherarh & Al-Masoudi & $\begin{array}{c}\text { Shoaib } \\
\text { Harimlae }\end{array}$ & Al-Khabiah \\
\hline & \multicolumn{4}{|c|}{ CFU X 10 } \\
\hline Control (bare soil) & 1.1 & 1.14 & 1.26 & 0.49 \\
\hline Tripleurospermum auriculatum & 16 & 1.8 & 16 & 16 \\
\hline Trigonella anguina & 16 & 9.2 & 5.4 & 1.54 \\
\hline Launaea capitata & 16 & 3.9 & 3.9 & 16 \\
\hline Rhayza stricta & 16 & 2.8 & 28 & 9.2 \\
\hline Hamada elegans & 5.4 & 3.5 & 16 & 16 \\
\hline Lycium shawii & 16 & 16 & 18 & 1.47 \\
\hline Acacia gerrardii & 16 & 5.4 & 35 & 2.8 \\
\hline Ziziphus nummularia & 16 & 16 & 28 & 16 \\
\hline Calotropis procera & 16 & 9.2 & 16 & 16 \\
\hline
\end{tabular}


Table.12 Counts of Streptomyces sp. in the rhizosphere of wild plants in some meadows of Riyadh region

\begin{tabular}{|c|c|c|c|c|}
\hline \multirow[t]{2}{*}{$\begin{array}{l}\text { Type of } \\
\text { plant }\end{array}$} & Al Kherarh & Al-Masoudi & $\begin{array}{c}\text { Shoaib } \\
\text { Harimlae }\end{array}$ & Al-Khabiah \\
\hline & \multicolumn{4}{|c|}{ CFU X $10^{3}$} \\
\hline Control (bare soil) & 2.4 & 1.3 & 1.5 & 7 \\
\hline Tripleurospermum auriculatum & 14 & 13 & 21 & 105 \\
\hline Trigonella anguina & 34 & 4 & 7 & 53 \\
\hline Launaea capitata & 5 & 5 & 9 & 102 \\
\hline Rhayza stricta & 12 & 17 & 4 & 74 \\
\hline Hamada elegans & 10 & 42 & 4 & 25 \\
\hline Lycium shawii & 30 & 24 & 13 & 50 \\
\hline Acacia gerrardii & 31 & 4 & 18 & 67 \\
\hline Ziziphus nummularia & 9 & 21 & 5 & 45 \\
\hline Calotropis procera & 95 & 30 & 25 & 30 \\
\hline
\end{tabular}

Table.13 Counts of cellulose decomposing bacteria in the rhizosphere of wild plants in some meadows of Riyadh region

\begin{tabular}{|c|c|c|c|c|}
\hline \multirow[t]{2}{*}{ Type of plant } & Al Kherarh & Al-Masoudi & $\begin{array}{c}\text { Shoaib } \\
\text { Harimlae }\end{array}$ & Al-Khabiah \\
\hline & \multicolumn{4}{|c|}{ CFU X $10^{3}$} \\
\hline Control (bare soil) & 1.93 & 1.2 & 1.4 & 3.2 \\
\hline Tripleurospermum auriculatum & 28 & 28 & 150 & 160 \\
\hline Trigonella anguina & 22 & 11 & 18 & 14 \\
\hline Launaea capitata & 5.4 & 9.3 & 33 & 160 \\
\hline Rhayza stricta & 93 & 1.7 & 28 & 160 \\
\hline Hamada elegans & 2.4 & 3.3 & 17 & 28 \\
\hline Lycium shawii & 3.9 & 3.9 & 16.2 & 18 \\
\hline Acacia gerrardii & 3.9 & 1.7 & 35 & 11 \\
\hline Ziziphus nummularia & 20.8 & 2.6 & 13.5 & 54 \\
\hline Calotropis procera & 98 & 1.7 & 160 & 22 \\
\hline
\end{tabular}

\section{Total microbial counts}

The densities of total microbial counts in the rhizosphere of different wild plants in four meadows are presented in table (6). The results indicated renounced differences in total microbial counts in the rhizosphere of various wild plants under selected four meadows of Riyadh region. Densities of total microbial counts were generally higher in rhizosphere of different wild plants, compared to control (bare soil) in studied meadows. These results confirm those found by Sunantapongsuk (2003) and Koo et al., (2005), who reported that microbial populations and activities were higher in rhizosphere than outside of the rhizosphere. The rhizosphere microbial community is normally more diverse and active than that in the bulk soil (Smalla et al., 2001, Majjami, 2020).

Data also showed higher total microbial counts in the rhizosphere of Calotropis procera and Lycium shawii plants in Shoaib Harimlae meadow exceeded those found in the rhizosphere of other wild plants under different meadows. In general, the total microbial counts in the rhizosphere of various wild plants were always higher in Shoaib 
Harimlae meadow as compared to the other selected meadows (Table 6). The diversity of soil microbial communities fluctuate in response to alterations in the environmental conditions (Steele and Streit, 2006). On the other hand, Soil type and soil structure also influence the dynamics of rhizosphere microbial populations. Concerning the total microbial counts in the root regions of nine wild plants, regardless of meadow type, the total microbial counts in the rhizosphere regions of Calotropis procera, Hamada elegans, Lycium shawii and Rhayza stricta plants were relatively higher than those in the rhizosphere of the other tested wild plants.

These results coincided with those stated by Curl and Truelove (1985) who found that both the quantity and quality of root exudates vary between plant species. In addition, it is also recognized that different cultivars of the same species may vary in their root exudation patterns. The quality of compounds released by plant roots appears to strongly influence the bacterial composition and activity in the rhizosphere, as shown by the preference of certain bacteria for exudates of different plant roots (El-Makki, 2017).

\section{Total fungi}

The total counts of fungi in the rhizosphere of different wild plants in four meadows are given in table (7). The densities of total fungi were always higher in the rhizosphere of wild plants than those in the nonrhizosphere soil under selected meadows. The higher total fungi counts in the rhizosphere soil of different wild plants were observed in Ziziphus snummularia and Calotropis procera plants growing in Al-Masoudi meadow, while the lower total fungi counts were recorded in the rhizosphere of Trigonella anguina plant in Al-Khabiah meadow. Generally, the total number of fungi increased in the rhizosphere of Ziziphus snummularia, Calotropis procera and Tripleurospermum auriculatum plants, regardless of meadow type (Table 7). On the other hand, total fungi in the rhizosphere of different wild plants showed higher values in Al-Masoudi meadow as compared to the other selected meadows. It is worthy to state that the role of fungi in soil is extremely complex and is fundamental to the soil ecosystem (Bridge and Spooner, 2001). Soil fungi play an important role in nutrient cycling, plant health and development (Thorn, 1997).

\section{Azotobacter sp.}

The total number of Azotobacter sp.in the rhizosphere and nonrhizosphere regions of wild plants under four meadows are presented in table (8). Azotobactersp. occurred in higher densities in rhizosphere of Hamada elegans and Tripleurospermum auriculatum plants under Al-Khabiah and Al-Kherarh meadows, respectively. On the other hand, the lowest counts of Azotobacter sp. were observed in rhizosphere of Rhayza stricta andAcacia gerrardii plants under Al-Masoudi meadow (Table 8). Concerning the Azotobacter sp. counts in the root regions of nine wild plants, regardless of meadow type, the total number of Azotobacter sp.in the rhizosphere regions of Launaea capitata and Tripleurospermum auriculatum were higher than those in the rhizosphere of the other tested wild plants or nonrhizosphere soil. Azotobacter sp. can fix atmospheric nitrogen in plants without any symbiosis as free-living bacteria (Gupta et al., 2002). Generally, Azotobacter sp. counts in the rhizosphere of various wild plants were always higher in Al-Kherarh and Al-Khabiah meadows as compared to the other selected meadows. These results may be due to the differences between selected four meadows in the physical-chemical conditions that predominant in the rhizosphere of different wild plants. The effect of physical and chemical on microbial survival and activity in soil are well documented (Van Overbeek and Van Elsas, 1997). 


\section{Azospirillum sp.}

Counts of Azospirillum sp. in the rhizosphere of wild plants in some meadows of Riyadh region are given in Table (9). It is evident from the results that counts of Azospirillum sp. were always higher in the rhizosphere of tested wild plants is compared the nonrhizosphere soil under different meadows. The higher Azospirillum sp. counts in the rhizosphere soil of various wild plants were observed in Tripleurospermum auriculatum, Trigonella anguina, Hamada elegans and Acacia gerrardii plants growing in Shoaib Harimlae meadow, while the lower Azospirillum sp counts were recorded in the rhizosphere of Rhayza stricta plant under the same meadow.

The total number of Azospirillum sp. exceeded in the rhizosphere of Tripleurospermum auriculatum and Trigonella anguina plants, regardless of meadow type (Table 9). Stimulation of root exudation in the rhizosphere of plants has been shown to occur in the presence of freeliving bacteria such as Azospirillum spp. and Azotobacter spp. (Heulin et al., 1987). The root exudation of different plants depends considerably on the physiological state of the superficial root cells (Prikryl and Vancur, 1980) On the other hand, counts of Azospirillum sp. in the rhizosphere of different wild plants showed higher values in Shoaib Harimlae and Al-Masoudi meadows as compared the other selected meadows

\section{Phosphate dissolving bacteria}

The densities of phosphate dissolving bacteria in the rhizosphere of different wild plants in four meadows are presented in table (10). It is evident from the results that the total microbial counts recordeddifferences in the rhizosphere soil between tested wild plants under four meadows. Densities of phosphate dissolving bacteria were generally higher in rhizosphere of different wild plants, compared to control (bare soil) under selected four meadows. High proportion of phosphate dissolving bacteria is concentrated in the rhizosphere, and they are metabolically more active than from other sources (Vazquez et al., 2000). Data also showed that phosphate dissolving bacteria in the rhizosphere of Acacia gerrardii and Lycium shawii plants in Al-Khabiah and Al-Kherarh meadows, respectively exceeded those found in the rhizosphere of other wild plants under different meadows. Concerning the phosphate dissolving bacteria in the root regions of nine wild plants, regardless of meadow type (Table 10) phosphate dissolving bacteria in the rhizosphere regions of Lycium shawii, Acacia gerrardii and Calotropis procera plants were higher than those in the rhizosphere of the other tested wild plants. In general, phosphate dissolving bacteria in the rhizosphere of various wild plants were always higher in AlKherarh meadow as compared to the other selected meadows. Kim et al., (1998) stated that phosphate dissolving bacteria are ubiquitous with variation in forms and population in different soils.

\section{Pseudomonas sp.}

The total number of Pseudomonas sp. in the rhizosphere and nonrhizosphere regions of wild plants under four meadows are presented in table (11). Pseudomonas sp. occurred in higher densities in rhizosphere of Acacia gerrardii, Ziziphus nummularia and Rhayza stricta plants at Shoaib Harimlae meadow. On the other hand, the lowest counts of Pseudomonas sp. were observed in rhizosphere of Trigonella anguina and Lycium shawii plants under Al-Khabiah meadow. Concerning the Pseudomonas sp. counts in the root regions of nine wild plants, regardless of meadow type, the total number of Pseudomonas sp. in the rhizosphere regions of Ziziphus nummularia plants were higher than those in the rhizosphere of the 
other tested wild plants or nonrhizosphere soil. Pseudomonas sp. make up a dominant population in soil and rhizosphere and exert growth promoting influence on a variety of plant species on account of their strong competitive behaviour, colonization potential and sustainability (El-Makki, 2017). Generally, pseudomonas sp. counts in the rhizosphere of various wild plants were higher in ShoaibHarimlae meadow as compared to the other selected meadows.

\section{Streptomyces sp.}

Table (12) shows the counts of Streptomyces sp. in the rhizosphere of wild plants in some meadows of Riyadh region. Counts of Streptomyces sp. were higher in the rhizosphere of tested wild plants is compared the nonrhizosphere soil under different meadows. These results coincided with those stated by El-Makki. (2017), who found that actinomycetes, including Streptomyces sp., become increasingly more abundant in the rhizosphere of maturing plants because dependent on mobilization of organic matter present in the rhizosphere soil.

The higher Streptomyces sp. counts in the rhizosphere soil of various wild plants were observed in Tripleurospermum auriculatum and Trigonella anguina plants growing in ShoaibHarimlae meadow and Calotropis procera plant growing in Al-Kherarh meadow, while the lower Streptomyces sp. counts were recorded in the rhizosphere of Trigonella anguina and Acacia gerrardii plants growing in Al-Masoudi meadow as well as Rhayza stricta and Hamada elegans plants growing in ShoaibHarimlae meadow (Table 12). The total number of Streptomyces sp. exceeded in the rhizosphere of Calotropis procera and Tripleurospermum auriculatum plants, regardless of meadow type. On the other hand, counts of Streptomyces sp. in the rhizosphere of different wild plants showed higher values in Al-Khabiah meadow as compared the other selected meadows

\section{Cellulose decomposing bacteria}

The densities of cellulose decomposing bacteriain the rhizosphere of different wild plants in four meadows are presented in table (13). The cellulose decomposing bacteria recorded differences in the rhizosphere soil between tested wild plants under four meadows. The cellulolytic microbes occupy a broad range of habitats. Some are free living and rid the environment of plant polysaccharides by converting them to the simple sugars, which they assimilate (ElMakki, 2017). Densities of cellulose decomposing bacteria were always higher in rhizosphere of different wild plants, compared to control (bare soil) under selected four meadows. Data also show that cellulose decomposing bacteriain the rhizosphere of Tripleurospermum auriculatum, Launaea capitata and Rhayza stricta plants in AlKhabiah meadow and Calotropis procera plant in Shoaib Harimlae meadow were exceeded those found in the rhizosphere of other wild plants under different meadows. Concerning the cellulose decomposing bacteriain the root regions of nine wild plants, regardless of meadow type, cellulose decomposing bacteriain the rhizosphere regions of Tripleurospermum auriculatum, Rhayza stricta and Calotropis procera plants were higher than those in the rhizosphere of the other tested wild plants.

In general, cellulose decomposing bacteria in the rhizosphere of various wild plants were always higher in Al-Khabiah meadow as compared to the other selected meadows. The cellulose-decomposing bacteria include mesophilic and thermophilic strains, inhabiting a great variety of environments, including the most extreme with regard to temperature, pressure and $\mathrm{pH}$ (Payer et al., 2006). 


\section{References}

Abd El-Fattah, R.T., Abou-Zeid, A.M. aand Altalhi, A.D. (2011). Allelopathic effects of Artemisia princeps and Launae sonchoids on rhizospheric fungi and wheat growth. African Journal of Microbiology Research 5(4): 419-424.

Abd El-Hafez, A.M. (1966) Some studies on acid producing microorganisms in soil and rhizosphere with special reference to phosphate dissolvers. Ph.D. Thesis, Fac. Agric. Ein Shams University.

Abdel-Hafez, S.I.I. (1981). Halophilic fungi of desert soils in Saudi Arabia. Mycopathologia., 75: 75-80.

Abdel-Malek, U. and Ishac, Y.Z. (1968). Evaluation of methods used in counting Azotobacter J.Appl.Bact. 31, 269-275

Abou-Zeid, A.M. and Altalhi, A.D. (2011). Allelopathic effects of Artemisia princeps and Launae sonchoids on rhizospheric fungi and wheat growth. African Journal of Microbiology Research 5(4): 419-424.

Al- nohait, F., A., Saleh. (2015). Diversity and Activity of microorganisms in the rhizosphere of some wild plants in Riyadh region, King Saud University (PhD Thesis, unpublished).

Al- Shaharani, T. S. and Shetta, N. D. (2011) Evaluation of growth, nodulation and nitrogen fixation of two Acacia species under salt stress, World Applied Science Journal 13 (2): 256- 265.

Alshaharani, T.S., and N.D. Shetta. (2015). Phenotypic and biochemical characterization of root nodule bacteria naturally associated with woody tree legumes in Saudi Arabia. Journal of Environmental Biology 36:363.

Al-Ghamdi, A.A.M. and Jaist, H.M. (2012). Interaction between Arbuscular Mycorrhiza and Heavy Metals in the Rhizosphere and Roots of Juniperus procera. International J. of. Agric\& Biology 14: 69-74.

Altalhi AD (2004). Studies on rhizospheric bacteria and fungi of some plants in Taif area, Saudi Arabia. Egypt. J. Appl. Sci., 19: $164-176$

Amal A, Wafaa OM, Amer MF, Mohamed M, Nabil AH (2003). Biodiversity of diazotrophs associated to the plant cover of north Sinai desert. Arch. Agron. Soil Sci., 49: 683-705.

Berg, G., Roskot, N., Steidle, A., Eberl, L., Zock, A., Smalla, K., 2002. Plantdependent genotypic and phenotypic diversity of antagonistic rhizobacteria isolated from different Verticillium host plants. Applied and Environmental Microbiology 68, 3328-3338.

Bowen, G. D. and Rovira, A. D. (1999). The rhizosphere and its management to improve plant growth. In: Advances in Agronomy. Vol. 66; p. 1-102.

Curl, E.A. and Truelove, B. (1985). The Rhizosphere, Springer, New York

De Nobili, M., Contin, M., Mondini, C., Brookes, P.C., 2001. Soil microbial biomass is triggered into activity by trace amounts of substrate. Soil Biology and Biochem-istry 33, 1163-1170.

Demoling, F., Figueroa, D., Bddth, E., 2007. Comparison of factors limiting bacterial growth in different soils. Soil Biology and Biochemistry 39, 2485 -2495.

Dobereiner, J., Marriel, I. E., and Nery, M. (1976). Ecological distribution of Spirillum lipoferum Beijerinck. Canadian Journal of Microbiology, 22(10), 1464-1473.

Gee G.W. and J.W. Bauder. Particle size Analysis. In Methods of Soil Analysis. Part $1,3^{\text {rd }}$. Physical and Mineralogical Methods. Edited by Sparks, D. L. Soil Science Society of America and American Society of Agronomy, Madison, WI., (1996), 377-382.

George Estefan, Rolf Sommer, and John Ryan (2013). Methods of Soil, Plant, and Water Analysis: A manual for the West Asia and North Africa region George Estefan, Rolf Sommer, and John Ryan $3^{\text {rd }}$ edition. International center for Agriculture research in the dry areas 


\section{(ICARDA)}

George Estefan, Rolf Sommer, and John Ryan (2013). Methods of Soil, Plant, and Water Analysis: A manual for the West Asia and North Africa region George Estefan, Rolf Sommer, and John Ryan $3^{\text {rd }}$ edition. International center for Agriculture research in the dry areas (ICARDA)

Gomes, N.C.H., Heuer, H., Schonfeld, J., Costa, R., Odnca-Hagler, L., and Smalla, K. (2001). Bacterial diversity of the rhizosphere of maize (Zea mays) grown in tropical soil studied by temperature gradient gel electrophoresis. Plant Soil, 233: 167.

Govindasamy, V., Senthilkumar, M., Mageshwaran, V., Annapurna, K., 2009. Detection and characterization of ACC deaminase in plant growth promoting rhizobacteria. Journal of Plant Biochemistry and Biotechnology 18, 71 $-76$.

Grayston, S. J., Vaughan, D. and Jones, D. (1996). Rhizosphere Carbon Flows It Trees, in Comparison with Annual Plants the Importance of Root Exudation and Its Impact on Microbial Activity and Nutrient Availability, Appl. Soil Ecol. 5, 29-56.

Gupta, A., Meyer, J. M. and Goel, R., 2002, Development of heavy metal resistant mutants of phosphate solubilizing Pseudomonas sp. NBR14014 and their characterization. Curr. Microbio., 45: 323-327.

Heulin, T., Gukert, A., and Balandreau, J. (1987). Stimulation of root exudation of rice seedlings by Azospirillum strains: carbon budget under gnotobiotic conditions. Biol. Fertil. Soils, 4: 9.

Hinsinger, P. (1998). How do plant roots acquire mineral nutrients? Chemical processes in the rhizosphere, Adv. Agron., 64, 225.

Jones, D. L. and Darrah, P. R. (1994). Role of Root-Derived Organic Acids in the Mobilization of Nutrients from the Rhizosphere, Plant Soil 166, 247-257.
Jones, D.L. and Darrah, P.R. (1995). Influx and efflux of organic acids across the soilroot interface of Zea mays L. and its implications in rhizosphere C flow, Plant Soil, 173, 103.

Jones, D.L. and Darrah, P.R. (1996). Resorption of organic compounds by roots of Zea mays L. and its consequences in the rhizosphere III. Characteristics of sugar influx and efflux, Plant Soil, 178: 153.

Loppert, R.H. and M. Suarez. (1996). Carbonate and Gypsum. In Methods of Soil Analysis. Part3 Chemical Methods. Edited by Sparks et al., SSSA and ASA, Madison, WI., 437-474.

Majjami, A. Y. (2020). Chemical and Biological Indicators of Soil Quality in Organic andConventional Agriculture Systems in Al-Jouf province, Saudi Arabia. Ph.D thesis, King Saud University, Saudi Arabia.

Mandeel, Q.A. (2002). Microfungal community associated with rhizosphere soil of Zygophyllum qatarense in arid habitats of Bahrain. Journal of Arid Environments 50: 665-681.

Marschner, H. (1995). Mineral Nutrition of Higher Plants, 2nd ed., Academic Press, London.

Marschner, P., Crowley, D. and Rengel, Z. (2011). Rhizosphere interactions between microorganisms and plants govern iron and phosphorus acquisition along the root axis - model and research methods. Soil Biology and Biochemistry 43: 883-894.

Nelson, D.W. and L.E. Sommers (1996)..Total carbon, organic carbon, and organic matter In: Methods of Soil Analysis, Part 3, "Chemical methods". Edited by Sparks et al., SSSA, and Madison, WI, USA, (1996).

Neumann, G. (2007). Root exudates and nutrient cycling. In: Marschner, P., Rengel, Z. (Eds.), Nutrient Cycling in Terrestrial Ecosystems. Springer, Berlin, pp. $123-158$.

Okon, Y. (1985). Azospirillum as a potential 
inoculant for agriculture. Trends in Biotechnology, 3: 223-228.

Page, A.L.; R.H.Miller and D.R.Keeney 1982. Methods of Soil Analysis. Part 2, No.9.in the Series Agronomy Amer. Soc. of Agren. Madison, Wis., USA.

. Prescott, L., Harley, J. and Klein, D. A. 1999. Microbiology. Boston: Mc- Graw-Hill. $962 \mathrm{p}$.

Rainwater F.H. and L.L. Thatcher. 1979. Methods of collection and analysis of water samples. Geo. Sur. Water supply. Paper No. 1454, Washington.

Rhodes, J.D. (1996). Salinity, Electrical conductivity and total dissolved solids. p. 417-435. In: Methods of Soil Analysis, Part 3, "Chemical methods". Edited by Sparks, SSSA, and Madison, WI, USA,

Sands, D. C. and Rovira, H. D. (1070). Isolation of fluorescent Pseudomonus with a selective medium. J. Appl. Microbiol. 20. 513-514.

Sheikh, H.M.A. (2010). Antimicrobial activity of certain bacteria and fungi isolated from soil mixed with human saliva against pathogenic microbes causing dermatological diseases. Saudi Journal of Biological Sciences 17: 331-339.

Shone, M.G.T. and Flood, A.L. (1983). Effects of periods of localised water stress on subsequent nutrient uptake by barley roots and their adaptation to osmotic adjustment, New Phytologist, 94: 561.

Sparks D.L., A. L. Page,, R. H Miller and D. R., Keeney (1996). Methods of soil analysis: Chemical methods, part 3, 3rd Edition. American Society of Agronomy and soil science society of America, Madison, WI.

Steele, H.L. and Streit, W.R. (2006). Metagenomics for the study of soil microbial communities. In: Cooper, J.E. and Rao, J.R. Molecular approaches to soil, rhizosphere and plant microorganisms analysis. $\mathrm{CAB}$ International, UK.

Sunantapongsuk, V., 2003. Study on soil microbial biodiversity in the rhizosphere of vetiver grass. J. Soil Water Conserv. 18 (3), 28-44.

Thomas, G.W. (1996) Soil pH and soil acidity.. In: Methods of Soil Analysis, Part 3. Chemical Methods. Edited by Sparks et al., Soil Science Society of America, Madison, WI, USA., 475-490.

Vaddar, U.B. (2007). Studies on grape rhizosphere microorganisms. M.Sc. Thesis, University of Agricultural Sciences, Dharwad.

Van Dongen, B.E., Schouten, S., and Sinnoghe Damsté, J.S. (2001). Gas chromatography/combustion/isotoperatio -monitoring mass spectrometric analysis of methylboronic derivatives of monosaccharides: a new method for determining natural ${ }^{13} \mathrm{C}$ abundance of carbohydrates, Rapid Commun. Mass Spectrom., 15: 496.

Vega, n.w.o. (2007). A review on beneficial effects of rhizosphere bacteria on soil nutrient availability and plant nutrient uptake. Rev. Fac. Nal. Agr. Medellín. 60(1): 3621-3643.

\section{How to cite this article:}

Fahad Nasser AL-Barakah, Fahd Abdulla Saleh Alnohait, Muhammad Sohaib Muhammad Ramzan and Samir Radwan. 2020. Microbial Diversity in the Rhizosphere of Some Wild Plants in Riyadh Region, Saudi Arabia. Int.J.Curr.Microbiol.App.Sci. 9(10): 3518-3534. doi: https://doi.org/10.20546/ijcmas.2020.910.406 